\title{
Egg Yolk Antibodies and Nanotechnology Applied to Animal Health and Production
}

\author{
Romina Bellingeri*, Picco N, Alustiza F, Grosso MC and Vivas A \\ Laboratorio de Biotecnología Animal, Argentina \\ *Corresponding author: Romina Bellingeri, Laboratorio de Biotecnología Animal, Facultad de Agronomía y Veterinaria, Córdoba, Argentina
}

Submission: 覀 April 20, 2018; Published: 眥 May 29, 2018

Keywords: Nanotechnology; Interdisciplinary science; Electronics; Material science; Health science

\section{Introduction}

Nanotechnology is a rapidly developing area of science and technology, which deals with the application, production and processing of materials with sizes less than 100nm (a nanometer being one billionth of a meter). In addition, it is an interdisciplinary science comprising of various disciplines such as physics, chemistry, electronics, material science, health science, biology, and veterinary science, and it has impact on many industries [1]

The ability to manufacture and manipulate material at nanoscale has offered opportunities to interface biological systems with outer world in new ways and with unprecedented precision. These emerging technologies have indicated great potential in functional foods and nutraceuticals for delivering bioactive compounds in functional foods to improve human and animal health [2,3]. Nanoscience and engineering can offer significant improvements in food safety, packaging and ultimately, novel food ingredients, because nanotechnology may create different and new properties [4]. Applications of nanotechnology in the food industry have been developing more slowly than other areas because of their complex structures and sensitivity. Some applications in the food industry are improving the uptake, absorption, and bioavailability of nutrients and supplements in the body, producing new or improved tastes, and textures of foods, improving food-packaging materials, developing nanosensors that can give information about the freshness or spoilage of products during transportation, storage of food products [5].

In this section, general particularities and applications of nanotechnology will be discussed with a special focus in nanocomposites for oral administration of IgY.

Nanomedicine for Therapeutics and Drug Delivery of IgY

Avian IgY antibodies have many attractive advantages over IgG in biochemical, immunological and production applications.
Recent advances in the use of IgY and nanomedicine shows good properties in diagnostic applications. Egg yolk antibodies have been absorbed onto gold nanoparticles to make nano goldIgY conjugates with possible uses in membrane isolation and nanotherapeutics [6]. Also, in immunehistochemistry, Xiao \& Gao [7] have shown that combining the high sensitivity and specificity of IgY and the high brightness and photo stability of quantum dots can improve detection and quantitation of biomarkers. In another study, homogeneous immunoassay for human IgG using oriented hen egg IgY immobilized on gold sol nanoparticles has been presented with a wide detection range and a limit of detection of 30ng.mL-1 of human IgG [8].

In animal production, the oral administration of $\operatorname{IgY}$ as prophylaxis or passive immmunization requires considerations about its stability to gastric $\mathrm{pH}$, proteolytic degradation and heat stability due to technological processes that are involved in the industrial processing of IgY. Therefore, to use the IgY as passive immunotherapy, it is necessary to find an effective method for preserver the biological function and therapeutic efficacy of this antibody during gastric passage.

Various techniques of micro and nano-encapsulation have been developed to protect IgY of the gastric inactivation. Shimizu et al. [9] reported that liposomal membrane prepared from egg yolk lecithin was almost completely stable against digestion by pepsin. The encapsulation of IgY, using enteric-coated gelatin capsules, has also been examined, and was found to significantly improve antibody stability [10]. An in vivo study using pigs as a model digestion, a coating of IgY-containing granules with a $\mathrm{pH}$-sensitive methacrylic acid copolymer was found to protect IgY from gastric inactivation [11]. Cho et al. [12] showed that two different emulsification methods involving multiple emulsification and heat gelation were for preparation of whey protein-based microcapsules containing immunoglobulin in yolk (IgY) were effective in protecting IgY from highly acidic conditions and heat treatment processes. The 
enhanced stability of IgY under harsh conditions and IgY storage stability were useful for applications in the food processing industry.

Other report described the use of microencapsulated and liposomes to protect IgY degradation. Microencapsulation with $10 \%$ or $20 \%$ b-cyclodextrin (b-CD) and arabic gum by a spray-drier was effective in protecting the IgY activity against pepsin. Liposome prepared at the lecithin/cholesterol ratio of $1 / 0.25$ (mole/mole) displayed satisfactory encapsulation efficiency (69\%) of IgY. Increase in cholesterol content in the liposomal structure exhibited a stronger protection effect of IgY against pepsin and acid [13]. On the other hand, Torché et al. [14] used PLGA-MS (poly (D, L-lactideco-glycolide)microspheres) to administrated locally (surgical technique), into the duodenum, an immunogenic protein (IgY) to evaluate systemic immune response in SPF Large White pigs. They proposed PLGA MS as a potential oral delivery system for antigen. Otherwise, Li et al. $[15,16]$ reported the use of chitosan-alginate microcapsules to protect IgY anti- K88+ ETEC (enterotoxigenic E. coli)-induced diarrhea using an in vivo pig model. Chitosanalginate microspheres could effectively protect the IgY antibodies from gastric inactivation after oral administration, resulting in an enhanced protection against ETEC-induced diarrhea in older pigs. Since the protein- and peptide-based drugs were highly susceptible to the gastrointestinal environments, some efforts were put on this area. Also, sucralfate, and anti-peptide ulcer agent, was evaluated to protect IgY (anti-H. pylori VacA) by oral preparations which can tolerate acid and pepsin. Over 30\% sucralfate enhanced the tolerance of IgY to low $\mathrm{pH}$ value and pepsin and the anti-freeze thawing ability [17].

\section{In vitro study of IgY protection by nanocomposites and toxicity evaluation}

The rational design of nutraceuticals delivered by micro/ nanocomposites requires the understanding of the relationship between the structure of the protein and their functional properties. Also it is necessary to understand the impact of the encapsulation system on the stability and the controlled liberation of the protein of interest on the targeted site.

As shown previously, a wide variety of micro/nanocomposites based delivery system has been described for the encapsulation of IgY. Recently, a new approach for the protection of IgY in simulated gastric conditions has been described. First, an anti-Escherichia coli IgY was obtained and characterized [18]. Then, intelligent hydrogels with ph-sensitive properties were synthesized and used to encapsulate the anti-Escherichia coli IgY.

The capacity of these structures to load, protect and release this IgY and the interaction between the IgY and hydrogels by Fourier Transform Infrared (FT-IR) spectroscopy were evaluated. The particle size and swelling percentage of the hydrogels were highly dependent on the $\mathrm{pH}$ of the buffer solution and the hydrogels could efficiently incorporate IgY and protect the IgY from simulated gastric conditions. However, IgY was slowly released at basic $\mathrm{pH}$ and a high percentage remained inside. The IR spectra showed that IgY interacts with the hydrogel in its network with extended hydrogen bonds [19].

The intelligent hydrogels were then improved and composite materials based on hydrogels and Carbon NanoTubes (CNT) were generated. For the synthesis, first oxidized CNT were wrapped with Chitosan (CH) and analyzed by FT-IR. Studies with Transmission Electron Microscopy (TEM) could confirm a CH layer lying around CNT. Chitosan wrapped CNT were then incorporated to the intelligent hydrogels for generate nanocomposites for protection and delivery of IgY. Swelling behavior in buffers at different $\mathrm{pH}$ revealed a significantly lower swelling when nanocomposites were exposed to an acid buffer solution. The mechanical properties of this delivery system were evaluated by measurements of elasticity and the hydrogels containing CNT-CH showed more resistance. The incorporation and liberation of IgY from hydrogel-CNT-CH were also assessed revealing an improved performance compared with the pure hydrogel. Hydrogel nanocomposites have been used in numerous biomedical applications and remote controlled drug delivery systems. However, only few studies have been reported so far on the biocompatibility of hydrogel-CNT-CH nanocomposites. The evaluation of the effect of these nanocomposites on cellular red ox balance of intestinal cells shows that the composites induce no oxidative stress. Cytotoxicity and apoptosis were also evaluated and there were no evidence of cytotoxicity or cell death. These preliminary findings are suggesting that hydrogelCNT-CH nanocomposites show improved properties and good biocompatibility in vitro making these biomaterials promising systems for IgY delivery purposes [20].

\section{Nanotechnology for Bovine Production Applications}

The use of the nanotechnology to veterinary medicine provides the opportunity for improving drug, molecules, and vaccine delivery and for bulk application to extensive production systems. However, there are still very limited the in vivo studies of nanotechnology applied to animals. Little investigations have been evaluated the application of nanoparticle-based therapeutics in bovines, among which may be mentioned: liposome for intramammary administration of streptomycin and gentamicin, liposome for intravenous application of adriamycin, niosome for intravenous application of flurbiprofen, liposome for transdermal administration of diclofenac [21].

Passive immunization using specific IgY antibodies has been previously studied in bovine, and its application has been carried to different objectives: protection against E. coli K99+ infection, reduction of E. coli 0157:H7 fecal shedding in feedlot steer, growth inhibition, internalization, and uptake by macrophages of E. coli 0111. Protection against Bovine Rotavirus-induced diarrhea in neonatal calves, reduction of morbidity and mortality due to respiratory pathogens in calves also has been described. Finally, the reduction of ruminal counts of Fusobacterium necrophorum and Streptococcus bovis, and reduction of symptoms in clinical and experimental mastitis caused by Staphylococcus aureus was reported [22] 
Previous IgY encapsulation methods has been shown a marked effect on the protection of this antibody from degradation under physiological digestive conditions, allowing its oral administration. Recently, it has been investigated the oral administration of IgY into previously described hydrogel-CNT-CH composites for the prevention of calf neonatal diarrhea caused by ETEC [18]. These delivery systems enhanced the efficiency of incorporation of IgY, allowed its controlled-time release and protected it of gastric inactivation. The administration of anti-ETEC IgY encapsulated into these nanocomposites, decreased the number of animals affected, the severity and duration of diarrhea. Necroses, atrophy of villous and inflammatory reactions were not observed in the intestinal mucosa of calves evaluated after administration of the nanocomposites. Significant differences in the percentage of degranulated and non-degranulated mast cells were not observed in any of the studied calves. Therefore, hydrogel-CNT$\mathrm{CH}$ nanocomposites could be used as an effective vehicle for oral administration of IgY in passive therapies.

\section{Nanotechnology for Porcine Production Applications}

Porcine production is in increasing growing and new technologies application. As principal problems derived from overcrowding there may be mentioned stress and health diseases. Swine neonatal diarrhea is one of the major causes of death and economic losses in swine production and the main etiological agent is Entero Toxigenic Escherichia coli (ETEC) around of world [23,24]. These problems have led producers to carry out breeding practices under strictly controlled sanitary conditions. However, not all these practices are enough to achieve ideal sanitary conditions, mainly due to the environmental survival capacity of E. coli strains. Moreover, extended use of antibiotic generates a considerable amount o resistance [25]. As an alternative of antibiotics emerged the egg yolk immunoglobulin (IgY). Oral administration of IgY, as a passive immunization strategy, present a promising approach for human and animals [26]. The successful use of IgY in pigs was reported previously by Kovacs-Nolan \& Mine [22].

The use of the nanotechnology applied to porcine delivery of different drugs has been reported. Little investigations have been evaluated the application of nanoparticle-based therapeutics in porcine, among which may be mentioned: dendrimers as vaccine adjuvants to prevent foot and mouth disease, liposome to delivery of vitamin $\mathrm{E}$ and polymeric particles to delivery of E. coli fimbriae as vaccine [21].

Recently, it has been investigated the oral administration of IgY into hydrogel-CNT-CH nanocomposites as a promising strategy of IgY delivery on intestinal tract. It was found that protected IgY was effective for the prevention of neonatal porcine diarrhea caused by ETEC. Neither symptoms nor signs of diarrhea disease were observed on piglets challenged and treated with IgY administered by hydrogel-CNT-CH nanocomposites, compared with challenged not-treated and challenges-treated with free IgY animals [23]. Nanotechnology shows to improve the efficiency of IgY to prevent piglet diarrhea, without toxic effects.

\section{Concluding Remarks and Future Trends}

Diarrhea in young animals is one of the most important diseases that affect livestock industry and continuing to cause high economic losses worldwide due to increased mortality, medication costs and decreased weight gain. A recent analysis has demonstrated the beneficial effect of IgY in controlling and preventing the diarrhea in domesticated animals. This supports the opinion that IgY is useful for prophylaxis and treatment of gastrointestinal infection by oral passive immunization as an alternative strategy to antibiotics. Hydrogel-CNT-CH nanocomposites show efficiently incorporate the anti-E. coli IgY. Despite the incomplete release of the therapeutic agent, protected IgY could improve the health status of E. coli challenged piglets and calves. Due the similarity between pigs and humans in terms of anatomy, genetics, and physiology the results obtained with hydrogel-CNT-CH nanocomposites can help to solve an important disease in Argentine and worldwide such us hemolytic-uremic syndrome. Additional studies will be necessary to improve several aspects like liberation rate and scalability.

The conjunction of IgY technology and nanotechnology has the capacity to offer innovative biosensors to applied in human and veterinary medicine. Recently, several articles have been published using IgY for immune diagnostic since it high sensitivity, specificity showing very interesting IgY capturing capabilities.

Although there are gaps that need to be filled, veterinary nanotechnology holds a great key in diagnostics and therapeutics of animal diseases and research is being intensified to breach the gaps.

\section{References}

1. Yeagle J (2007) Nanotechnology and the FDA, Virginia Journal of Law \& Technology, University of Virginia, 12, 6.

2. Hill EK, Li J (2017) Current and future prospects for nanotechnology in animal production. Journal of Animal Science and Biotechnology 8(1): 26.

3. Manuja A, Kumar B, Singh R (2012) Nanotechnology developments: opportunities for animal health and production. Nanotechnology Development 2(1).

4. Scott N, Chen H (2013) Nanoscale Science and Engineering for Agriculture and Food Systems. Industrial Biotechnology 9: 17-18.

5. Otles S, Yalcin B (2013) Food Chemestry and Nanoscience, Journal of Nanomaterials and Molecular Nanotechnology 2.

6. Gupta D, Kumar D, Dwivedi M, Tripathi V, Phadke-Gupta P, et al (2013) Nanophosphors-Nanogold Immunoconjugates in Isolation of Biomembranes and in Drug Delivery. In: Mishra AK, (Ed.) Nanomedicine for Drug Delivery and Therapeutics. John Wiley \& Sons, Inc., Hoboken, NJ, USA.

7. Xiao Y, Gao X (2010) Use of IgY antibodies and semiconductor nanocrystal detection in cancer biomarker quantitation. Biomark Med 4 (2): 227-239.

8. Yeritsyan HE, Gasparyan VK (2012) Homogeneous immunoassay for human IgG using oriented hen egg IgY immobilized on gold sol nanoparticles. Microchimica Acta 176(1-2): 117-122.

9. Shimizu M, Miwa Y, Hashimoto K, Goto A (1993) Encapsulation of chicken egg yolk immunoglobulin G (IgY) by liposomes. Bioscience, Biotechnique and Biochemestry 57(9): 1445-1449. 
10. Akita E, Nakai S (2000) Preparation of enteric-coated gelatin capsules of IgY with cellulose acetate phthalate. In Egg Nutrition and Biotechnology: Publishing Press: Wallingford, UK, pp 301-310.

11. Kovacs-Nolan J, Mine Y (2005) Microencapsulation for the gastric passage and controlled intestinal release of immunoglobulin Y. J Immunol Methods 296(1-2): 199- 209.

12. Cho YH, Lee JJ, Park IB, Huh CS, Baek YJ, et al. (2005) Protective effect of microencapsulation consisting of multiple emulsification and heat gelation processes on immunoglobulin in yolk. Journal of Food Science 70(2): E148-E151.

13. Chang HM, Lee YC, Chen CC, Tu YY (2002) Microencapsulation protects immunoglobulin inyolk (IgY) specific against helicobacter pylori urease. Journal of Food Science 67(1): 15-20.

14. Torché AM, Le Dimna M, Le Corre P, Mespléde A, Le Gal S, et al. (2006) Immune responses after local administration of IgY loaded-PLGA microspheres in gut-associated lymphoid tissue in pigs. Veterinary Immunology and Immunopathology 109(3-4): 209-217.

15. Li XY, Jin LJ, McAllister T, Stanford K, Xu J, et al. (2007) Chitosan-alginate microcapsules for oral delivery of egg yolk immunoglobulin (IgY). J Agric Food Chem 55(8): 2911-2917.

16. Li XY, Jin LJ, Uzonna JE, Li SY, Liu JJ, et al. (2009) Chitosan-alginate microcapsules for oral delivery of egg yolk immunoglobulin (IgY): in vivo evaluation in a pig model of enteric colibacilosis. Vet Immunol and Immunopathol 129(1-2): 132-136.

17. Xun Z, Li-Yuan G, Zhibang Y, Xiaoping C (2010) Protective effects of sucralfate on anti-H. pylori VacA IgY in vivo and in vitro. African Journal of Microbiology Research 4(11): 1091-1099.

18. Bellingeri R, Busso L, Alustiza F, Picco N, Molinero D, et al. (2013) Characterization of egg yolk Immunoglobulin (IgY) against enterotoxigenic Escherichia coli and evaluation of its effects on bovine intestinal cells. African Journal of Microbiology Research 7(5): 398-405.
19. Bellingeri RV, Picco NY, Alustiza FE, Canova JV, Molina MA, et al. (2015)a $\mathrm{pH}$-responsive hydrogels to protect IgY from gastric conditions: In vitro evaluation. J of Food Sci and Technol 52(5):3117-3122.

20. Bellingeri R, Alustiza F, Picco N, Acevedo D, Molina MA, et al. (2015)b In Vitro Toxicity Evaluation of Hydrogel-Carbon Nanotubes Composites on Intestinal Cells. Journal of Applied Polymer Science 132(5).

21. Underwood C, van Eps AW (2012) Nanomedicine and veterinary science: the reality and the practicality. Vet J 193(1):12-23.

22. Kovacs-Nolan J, Mine Y (2012) Egg yolk antibodies for passive immunity. Annual Review of Food Science and Technology 3:163-182.

23. Alustiza F, Bellingeri R, Picco N, Motta C, Grosso MC, et al. (2016) IgY against enterotoxigenic Escherichia coli administered by hydrogelcarbon nanotubes composites to prevent neonatal diarrhoea in experimentally challenged piglets. Vaccine 34(28): 3291-3297.

24.Vu-Khac H, Holoda E, Pilipcinec E, Blanco M, Blanco JE, et al. (2007) Serotypes, virulence genes, intimin types and PFGE profiles of Escherichia coli isolated from piglets with diarrhoea in Slovakia. Vet J 174(1): 176-187.

25. Docic M, Bilkei G (2003) Differences in antibiotic resistance in Escherichia coli, isolated from East-European swine herds with or without prophylactic use of antibiotics. J Vet Med B infect Dis Vet Public Health 50(1): 27-30.

26. Diraviyam T, Zhao B, Wang Y, Schade R, Michael A, et al. (2014) Effect of Chicken Egg Yolk Antibodies (IgY) against Diarrhea in Domesticated Animals: A Systematic Review and Meta-Analysis. PLoS one 9(5): e97716.

27. Picco N (2013) Tecnología de anticuerpos aviarios y nanotecnología aplicada a la sanidad bovina. Doctoral Thesis, Biblioteca Juan Filloy, Universidad Nacional de Río Cuarto. Río Cuarto, Córdoba, Argentina.
Creative Commons Attribution 4.0 International License

For possible submissions Click Here

\section{Submit Article}

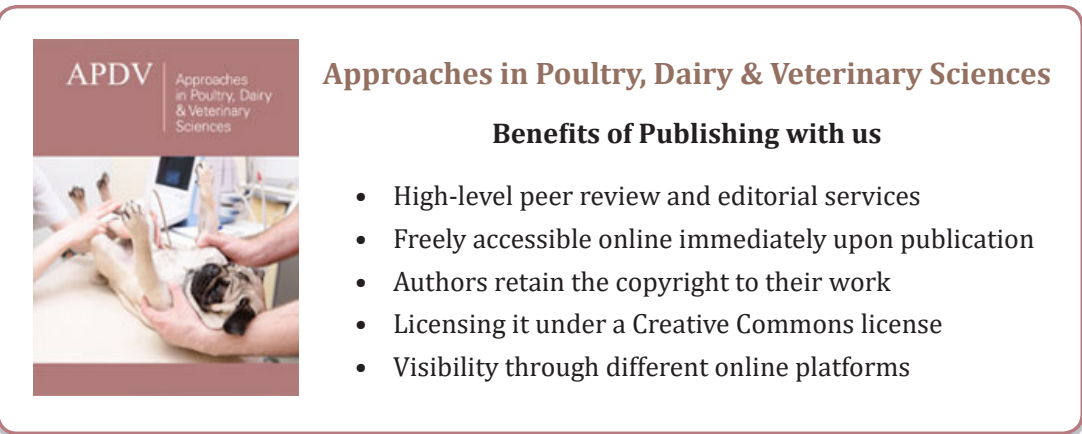

